\title{
ON THE BASIC CHARACTER OF RESIDUE CLASSES
}

\author{
P. HILTON, J. HOOPER AND J. PEDERSEN
}

\begin{abstract}
Let $t, b$ be mutually prime positive integers. We say that the residue class $t \bmod b$ is basic if there exists $n$ such that $t^{n} \equiv-1 \bmod b$; otherwise $t$ is not basic. In this paper we relate the basic character of $t \bmod b$ to the quadratic character of $t$ modulo the prime factors of $b$. If all prime factors $p$ of $b$ satisfy $p=3 \bmod 4$, then $t$ is basic mod $b$ if $t$ is a quadratic nonresidue $\bmod p$ for all such $p$; and $t$ is not basic $\bmod b$ if $t$ is a quadratic residue $\bmod p$ for all such $p$. If, for all prime factors $p$ of $b, p \equiv 1 \bmod 4$ and $t$ is a quadratic non-residue mod $p$, the situation is more complicated. We define $d(p)$ to be the highest power of 2 dividing $(p-1)$ and postulate that $d(p)$ takes the same value for all prime factors $p$ of $b$. Then $t$ is basic mod $b$. We also give an algorithm for enumerating the (prime) numbers $p$ lying in a given residue class mod $4 t$ and satisfying $d(p)=d$. In an appendix we briefly discuss the case when $b$ is even.
\end{abstract}

\section{0 . Introduction}

In a series of papers [1,through 4], culminating in the monograph [5], Hilton and Pedersen developed an algorithm - in fact, two algorithms, one being the reverse of the other - for calculating the quasi-order of $t \bmod b$, where $t, b$ are mutually prime positive integers, and determining whether $t$ is basic mod $b$. Here the quasi-order of $t \bmod b$ is the smallest positive integer $k$ such that $t^{k} \equiv \pm 1 \bmod b$; and $t$ is said to be basic if, in fact, $t^{k} \equiv-1 \bmod b$. Thus $t$ is basic if and only if the order of $t \bmod b$ is twice the quasi-order of $t$ mod $b$ (in the contrary case the quasi-order and the order coincide). Froemke and Grossman carried the number-theoretical investigation considerably further in [6] and drew attention to the importance, where $b$ is prime, of the quadratic character of $t \bmod b$ in their arguments.

Our object in this paper is to relate the basic character of $t \bmod b$ to the quadratic character of $t$ modulo the prime factors of $b$. We assume $b$ odd, but add a few remarks in an appendix on the case when $b$ is even.

Given a pair $(t, p)$ where $p$ is an odd prime not dividing $t$, we distinguish 4 possibilities as follows: may or may not be a quadratic residue mod $p$, and we may have $p \equiv 1 \bmod 4$ or $p \equiv 3 \bmod 4$. We restrict attention, in our 
discussion of the basic character of $t \bmod b$, to the situation in which all prime factors $p$ of $b$ place $(t, p)$ in the same class. If $t$ is a quadratic residue mod $p$ and $p \equiv 1 \bmod 4$, we are unable to draw any general conclusion about the basic character of $t \bmod p$. Thus, for example, $7^{7} \equiv 1 \bmod 29,7^{7} \equiv-1 \bmod 113$, and 7 is a quadratic residue modulo 29 or 113. If all prime factors $p$ of $b$ satisfy $p \equiv 3 \bmod 4$, it is easy to draw general conclusions about the basic character of $t \bmod b$; our results are given in Section 2 .

The most interesting case for our purposes is that in which $t$ is not a quadratic residue $\bmod p$ and $p \equiv 1 \bmod 4$, for all prime factors $p$ of $b$. It then becomes important to be able to calculate the function $d(p)$, where $d(p)=d$ if $p=$ $1+2^{d} c$, with $c$ odd. Thus $d$ is a positive integer and, in fact, $d \geq 2$ in the case we are discussing. Since the quadratic character of $t \bmod p$ depends only on the residue class of $p \bmod 4 t$, we give an algorithm for enumerating those primes $p$, as functions of $s$ and $d$, such that

$$
d(p)=d, \quad p \equiv s \bmod 4 t, \quad 1 \leq s \leq 4 t-3
$$

If the prime factors $p$ of $b$ are confined to those satisfying (0.1) for fixed $s, d$, then, as we show in Section $3, t$ is basic mod $b$.

In Section I we announce some elementary results which are used in proving our main theorems.

Throughout the paper we use the symbol $\epsilon$ for a number which is +1 or -1 .

\section{Some preliminary lemmas}

The first result extends to the quasi-order a farniliar result on order.

Lemma 1.1. Let the quasi-order of $t \bmod b$ be $n$ and let $t^{m} \equiv \epsilon \bmod b$. Then $n \mid m$.

Proof: Let $m=q n+r, 0 \leq r<n$, and $t^{n} \equiv \eta \bmod b, \eta= \pm 1$. Then $t^{r}=t^{m}\left(t^{n}\right)^{-q} \equiv \epsilon \eta^{q}= \pm 1 \bmod b$, so that $r=0$.

We now restrict $b$ by the condition $b \geq 3$, so that the basic character of $t$ mod $b$ comes into question.

Lemma 1.2. The residue $t \bmod b$ is basic if and only if $t^{m} \equiv-1 \bmod b$ for some exponent $m$.

Proof: The necessity of the condition is obvious. Suppose then that $t^{m} \equiv$ $-1 \bmod b$ and that the quasi-order of $t \bmod b$ is $n$. Then $n \mid m$, by Lemma 1.1. Thus, if $t^{n} \equiv 1 \bmod b_{1}$ it follows that $t^{m} \equiv 1 \bmod b$. This contradiction shows that $t^{n} \equiv-1 \bmod b$, so that the residue $t \bmod b$ is basic. 
Lemma 1.3. The residue $t \bmod b$ is non-basic if $t^{m} \equiv 1 \bmod b$ for some odd exponent $m$.

Proof: Let the quasi-order of $t \bmod b$ be $n$ with $t^{n} \equiv \epsilon \bmod b$. Then $n \mid m$, so that $m=n q$. Since $m$ is odd, $q$ is odd. Thus $t^{m} \equiv \epsilon^{q}=\epsilon \bmod b$, so $\epsilon=1$ and the residue $t \bmod b$ is non-basic.

Our next result is of a different kind.

Proposition 1.4. Let $x \equiv y \bmod m$. Then $x^{m^{k-1}} \equiv y^{m^{k-1}} \bmod m^{k}, k \geq 1$.

Proof: We argue by induction on $k$, the case $k=1$ being trivial. If we assume $x^{m^{k-1}} \equiv y^{m^{k-1}} \bmod m^{k}$ for a certain $k \geq 1$, then

$$
\begin{aligned}
x^{m^{k-1}} & =y^{m^{k-1}}+\lambda m^{k}, \text { so that } \\
x^{m^{k}} & =\left(y^{m^{k-1}}+\lambda m^{k}\right)^{m} \\
& =y^{m^{k}}+\lambda m^{k+1} y^{m^{k-1}(m-1)}+\left(\begin{array}{c}
m \\
2
\end{array}\right) \lambda^{2} m^{2 k} y^{m^{k-1}(m-2)}+\ldots \\
& \equiv y^{m^{k}} \bmod m^{k+1}
\end{aligned}
$$

This establishes the inductive step, and hence the proposition.

We have the immediate consequence:

Lemma 1.5. Let $c \equiv \epsilon \bmod m$, with $m$ odd. Then $c^{m^{k-1}} \equiv \epsilon \bmod m^{k}, k \geq 1$.

Proof: We have only to note that $\epsilon^{m^{k-1}}=\epsilon$ if $m$ is odd.

\section{The main results}

We recall the following key results on quadratic reciprocity.

Theorem 2.1 (Euler). Let $p$ be an odd prime. Then

(i) $t^{\frac{p-1}{2}} \equiv 1 \bmod p$ if and only if $t$ is a quadratic residue modp

(ii) $t^{\frac{p-1}{2}} \equiv-1 \bmod p$ if and only if $t$ is not a quadratic residue mod $p$.

Theorem 2.2 (Gauss). Let $p$ be an odd prime. Then the quadratic character of $t$ mod $p$ depends only on the residue class of $p$ mod $4 t$ and is the same for two odd primes $p$ and $q$ such that $p \equiv-q \bmod 4 t$. 
Thus, given $t$ and $p$, we distinguish 4 classes into which the pair $(t, p)$ may fall:

$I p \equiv 1 \bmod 4, t^{\frac{p-1}{2}} \equiv 1 \bmod p$;

II $p \equiv 1 \bmod 4, t^{\frac{r-1}{2}} \equiv-1 \bmod p$;

III $p \equiv 3 \bmod 4, t^{\frac{p-1}{2}} \equiv 1 \bmod p$;

IV $p \equiv 3 \bmod 4, t^{\frac{y-1}{2}} \equiv-1 \bmod p$;

We will say nothing further about residues $t \bmod b$ if $b$ admits a factor $p$ such that $(t, p)$ is in class I. We will henceforth, until otherwise stated, assume that $b$ is odd.

Theorem 2.3. Suppose that the prime factors $p$ of $b$ are all such that $(t, p)$ is in Class III. Then the residue $t$ is not basic mod $b$.

Proof: Let $b=\Pi_{i=1}^{N} p_{i}^{k_{i}}, k_{i} \geq 1$. Then $\frac{p_{i}-1}{2}$ is odd and $t^{\frac{p_{i}-1}{2}} \equiv 1 \bmod p_{i}$.

By Lemma $1.5, t^{\left(\frac{p_{i}-1}{2}\right) p_{i}^{k_{i}-1}} \equiv 1 \bmod p_{i}^{k_{i}}$. Set $m=\Pi_{i=1}^{N}\left(\frac{p_{i}-1}{2}\right) p_{i}^{k_{i}-1}$. Then $m$ is odd, and

$$
t^{m} \equiv 1 \bmod p_{i}^{k_{i}}
$$

It follows that $t^{m} \equiv I \bmod b$, so that, by Lemma 1.3 , the residue $t$ is not basic $\bmod b$.

Theorem 2.4. Suppose that the prime factors $p$ of $b$ are all such that $(t, p)$ is in Class IV. Then the residue $t$ is basic mod b.

Proof: We argue as for Theorem 2.3, except that now

$$
\begin{aligned}
t^{\left(\frac{p_{i}-1}{2}\right) p_{i}^{k_{i}-1}} & \equiv-1 \bmod p_{i}^{k_{i}}, \\
t^{m} & \equiv-1 \bmod p_{i}^{k_{i}}, \\
t^{m} & \equiv-1 \bmod b,
\end{aligned}
$$

with $m$ odd. We apply Lemma 1.2 to obtain the result.

Example 2.1. Let $t=7$. Then, by Theorem 2.2, we must consider primes $p$ mod 28 . We easily find

$$
\begin{aligned}
& p \equiv 1 \text { or } 27 \bmod 28: 7^{\frac{p-1}{2}} \equiv 1 \bmod p \\
& p \equiv 3 \text { or } 25 \bmod 28: 7^{\frac{p-1}{2}} \equiv 1 \bmod p \\
& p \equiv 5 \text { or } 23 \bmod 28: 7^{\frac{p-2}{2}} \equiv-1 \bmod p \\
& p \equiv 9 \text { or } 19 \bmod 28: 7^{\frac{p-1}{2}} \equiv 1 \bmod p \\
& p \equiv 11 \text { or } 17 \bmod 28: 7^{\frac{p-1}{2}} \equiv-1 \bmod p \\
& p \equiv 13 \text { or } 15 \bmod 28: 7^{\frac{p-1}{2}} \equiv-1 \bmod p
\end{aligned}
$$


Thus

$(7, p)$ is in Class I if $p \equiv 1,9,25 \bmod 28$;

$(7, p)$ is in Class II if $p \equiv 5,13,17 \bmod 28$;

$(7, p)$ is in Class III if $p \equiv 3,19,27 \bmod 28$;

$(7, p)$ is in Class IV if $p \equiv 11,15,23 \bmod 28$.

We conclude that 7 is not basic $\bmod b$ if $b$ is a product of primes $p$ such that $p \equiv 3,19$ or $27 \bmod 28$; and 7 is basic $\bmod b$ if $b$ is a product of primes $p$ such that $p \equiv 11,15$ or $23 \bmod 28$.

As we have said, no inference can be drawn if $b$ is a product of primes $p$ such that $p \equiv 1,9$ or $25 \bmod 28$. Indeed, the fact that $(7, p)$ is then in Class $I$ is a special case of the following phenomenon, which we describe herc for the sake of completeness.

Proposition 2.5. Let $p$ be an odd prime such that $p=k^{2}+4 l$. Then any factor of $l$ is a quadratic residue $\bmod p$.

Proof: It suffices to prove this for prime factors $q$ of $l$. Now if $q=2$, then $p \equiv 1 \bmod 8$, so 2 is a quadratic residue mod $p$. If $q$ is odd, then $p$ is a quadratic residue $\bmod q$ and $\frac{p-1}{2}$ is even, so that, by quadratic reciprocity, $q$ is a quadratic residue mod $p$.

Note that it follows, by Theorem 2.1 , that $7^{\frac{2-1}{2}} \equiv 1 \bmod p$ if $p \equiv 1,9$ or $25 \bmod 28$.

We will devote the next section to a discussion of the Class II. At this point, we are content to remark

Theorem 2.6. Suppose that $b=p^{k}$, where $(t, p)$ is in Class II. Then the residue $t$ is basic mod $b$.

Proof: $t^{\left(\frac{p-1}{2}\right) p^{k-1}} \equiv-1 \bmod p^{k}$. Apply Lemma 1.2 .

In the next section we generalize this obvious conclusion.

\section{The class II situation}

We define a function $d$ from positive integers $\geq 2$ to non-negative integers by

$$
d(n)=d \Leftrightarrow 2^{d} \mid(n-1), 2^{d+1} x(n-1) .
$$

Notice that, for an odd prime $p, d(p) \geq 1$ and that $d(p) \geq 2$ if $(t, p)$ is in Class II. Let $d$ be a fixed integer $\geq 2$; we then have the following theorem, generalizing Theorem 2.6. 
in this way, splitting the inequality $d(p) \geq d$ into the two possibilities $d(p)=d$ or $d(p) \geq d+1$. We demonstrate this tree in Figure 1 .

$\bmod$

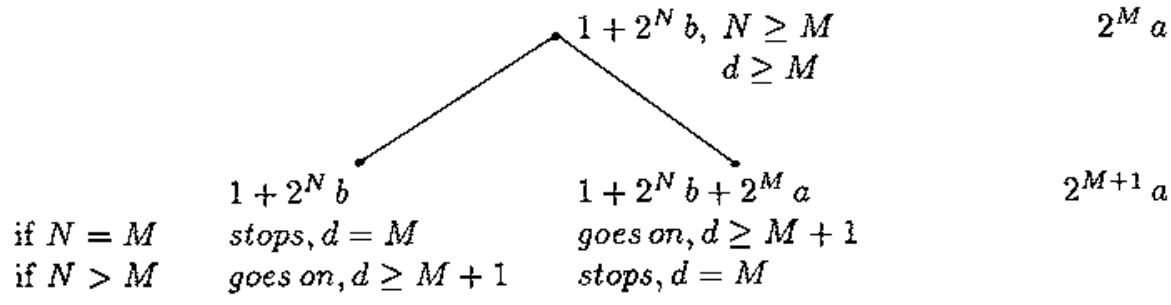

General Case

Figure 1

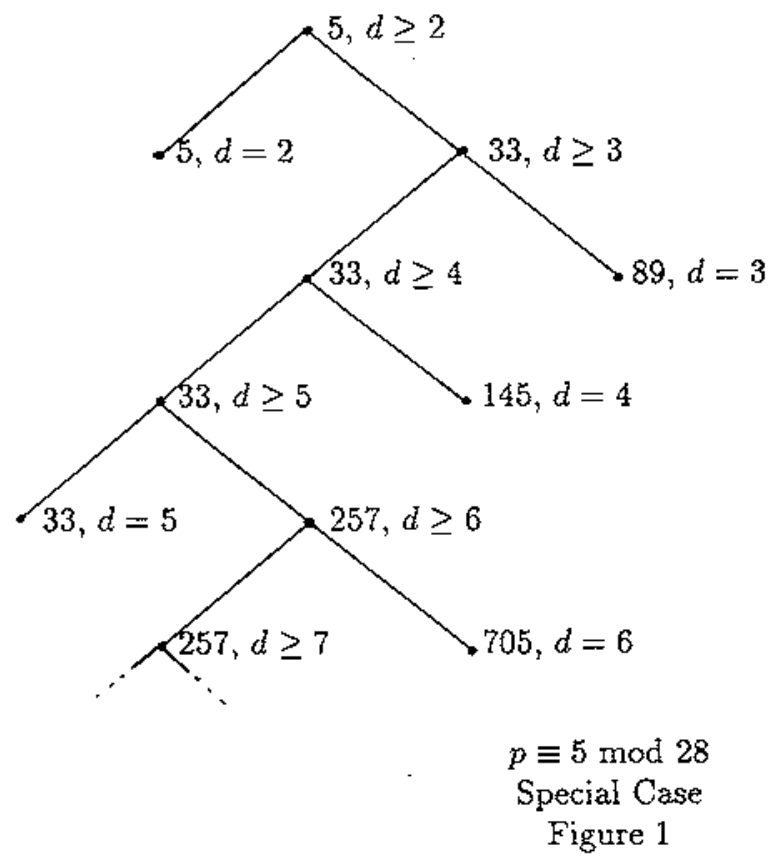

$\bmod$

The tree provides the conceptual basis for the proof (see Theorem 3.2) that the primes $p$ satisfying the congruence $p \equiv 2^{d} c+1 \bmod 2^{d+1} u$ constitute the totality of the primes $p$ satisfying $p \equiv s \bmod 4 t$ and $d(p)=d$. For we may argue by induction on $d$ that there is only one residue class mod $2^{d+1} u$ containing such primes. We assume $n \geq m+2$ and we rewrite (3.4) as

$$
2^{d} c(d) \equiv 2^{n} v \bmod u,
$$


to emphasize the dependence of $c$ on $d$; recall $d \geq m+2$. We start the induction (and the tree) by rewriting $p \equiv s \bmod 4 t$, using (3.11), as

$$
p \equiv 1+2^{m+2} c(m+2) \bmod 2^{m+2} u .
$$

Then (3.12) branches into the two congruences

$$
\left\{\begin{array}{l}
p \equiv 1+2^{m+2} c(m+2) \bmod 2^{m+3} u \\
p \equiv 1+2^{m+2} c(m+2) \pm 2^{m+2} u \bmod 2^{m+3} u
\end{array}\right.
$$

the sign being chosen so that the right-hand side is in the range $\left(0,2^{m+3} u\right)$. The first possibility in (3.13) yields $d(p)=m+2$ (recall that $c(d)$ is always odd) and the second yields $d(p) \geq m+3$, since $u$ is also odd. Thus our assertion holds in the initial case $d=m+2$.

Now assume inductively that, for $d(p) \geq d$, we require

$$
p \equiv 1+2^{d} c(d) \bmod 2^{d} u,
$$

for some $d \geq m+2$. Then, as above, we find that, for $d(p)=d$, we require

$$
p \equiv 1+2^{d} c(d) \bmod 2^{d+1} u ;
$$

while, for $d(p) \geq d+1$, we require

$$
p \equiv 1+2^{d} c(d) \pm 2^{d} u \bmod 2^{d+1} u .
$$

This shows the uniqueness of the residue class $\bmod 2^{d+1} u$ of $p$, given $d(p)=d$. But it also shows that, if $c(d+1)$ is to be odd, to satisfy the inequality $1 \leq$ $c(d+1) \leq 2 u-1$, and to render $p \equiv 1+2^{d+1} c(d+1) \bmod 2^{d+1} u$ equivalent to $(3.16)$, then $c(d+1)$ is determined by

$$
c(d+1)= \begin{cases}\frac{1}{2}(c(d)+u) \text { if } & \frac{1}{2}(c(d)+u) \text { is odd } \\ \frac{1}{2}(c(d)-u) \text { if } & \frac{1}{2}(c(d)-u) \text { is odd and positive } \\ \frac{1}{2}(c(d)+3 u) \text { if } & \frac{1}{2}(c(d)-u) \text { is odd and negative }\end{cases}
$$

Thus, in any case, $2 c(d+1) \equiv c(d) \bmod u$, so that, if $2^{d} c(d) \equiv 2^{n} v \bmod u$, then $2^{d+1} c(d+1) \equiv 2^{n} v \bmod u$. This establishes the inductive step and also gives us a recurrence relation (3.17) for determining $c(d)$. Of course, this recurrence relation is deducible from

$$
2 c(d+1) \equiv c(d) \bmod u
$$

which also shows why the period of $c(d)$ is the order of $2 \bmod u$.

We emphasize that $(3.17)$, together with the initial congruence $c(m+2) \equiv$ $2^{n-m-2} v \bmod u$, gives a practical algorithm for determining the values $c(d)$ 
-recall that $c(d)$ is odd with $1 \leq c(d) \leq 2 u-1$. We then apply Theorem 3.2 to determine the primes $p$ in a given residue class mod $4 t$ and satisfying $d(p)=d$.

Example 3.2. Let $t=11, s=5$, so that $p \equiv 5 \bmod 44$. Thus

$$
\begin{aligned}
& s=5, u=11, m=0 \\
& t=11, v=1, n=2
\end{aligned}
$$

To calculate $c(d)$ we start the induction with $c(2) \equiv 1 \bmod 11$, so $c(2)=1$. Now $l$, the order of $2 \bmod 11$, is 10 , so the period of $c(d)$ is 10 , and (3.17) yields the table

\begin{tabular}{|l|cccccccccc|}
\hline$d$ & 2 & 3 & 4 & 5 & 6 & 7 & 8 & 9 & 10 & 11 \\
$c$ & 1 & 17 & 3 & 7 & 9 & 21 & 5 & 19 & 15 & 13 \\
\hline
\end{tabular}

The tree diagram is shown in Figure 2.

$\bmod$

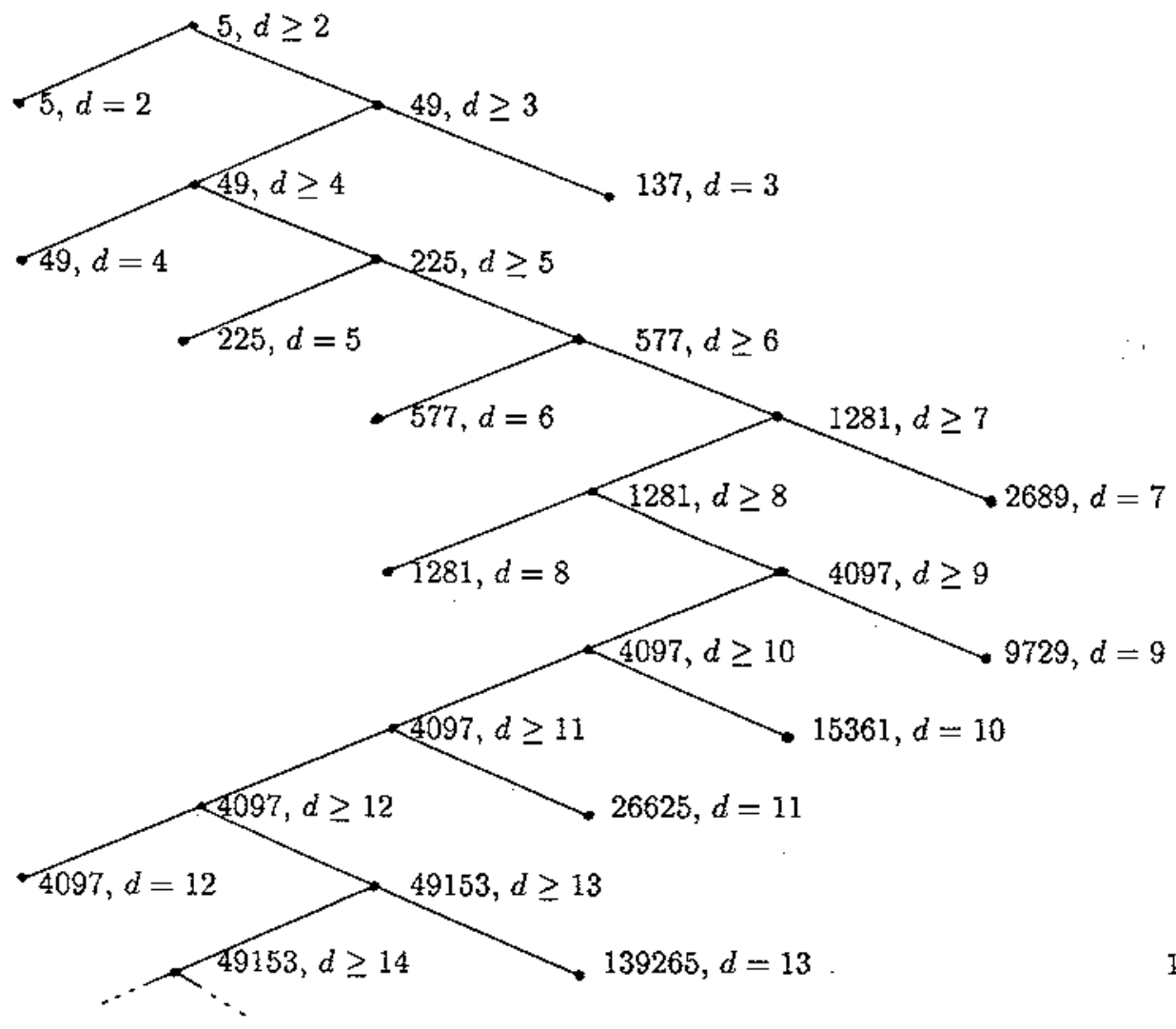

Figure 2

Notice that not only does $c(d)$ repeat from $d=12$ onwards, but that the whole tree pattern repeats. 


\section{Appendix}

We introduce the equivalence relation $t \sim t^{\prime}$ in the set of positive integers, defined by

$$
t \sim t^{\prime} \Leftrightarrow t / t^{\prime} \text { is the square of a rational number. }
$$

It is then plain that $t$ is a quadratic residue mod $p$ if and only if $t^{\prime}$ is a quadratic residue $\bmod p$ (where $t \sim t^{\prime}$ and $t, t^{\prime}$ are both prime to $p$ ). This shows that the class, in the sense of Section 2, to which $(t, p)$ belongs depends only on the equivalence class of $t$. Thus our conclusions embodied in Theorems 2.3, $2.4,2.6$ and 3.1 apply to entire equivalence classes of integers $t$. Moreover, it follows that, insofar as we only exploit these results, we may assume that $t$ is square-free. This has the important effect that, in applying the techniques of Section 3 to provide an explicit description of those primes $p$ such that $(t, p)$ is in class II and $d(p)=d$, we may, in practice, confine attention to $m=0$ or 1 . Of course, the formal analysis for $t=12$, say, is different from that for $t=3$, but the conclusions are coextensive-and the same!

Indeed, so far as the methods of this paper are concerned, we may really confine ourselves to the case that $t$ is itself a prime. For it is trivial to derive the quadratic character of $t$ from the quadratic characters of its prime factors; and our deductions are exclusively based on the quadratic character of $t$ modulo the prime factors of $b$. Notice that we are far from saying that the basic character of $t \bmod b$ can be deduced from that of the prime factors of $t$-just as we do not clain that, in general, the basic character of $t \bmod b$ depends only on the equivalence class of $t$ under the equivalence relation (4.1). For example, 4 is basic mod 17 but 1 is not. It remains to make a remark if $b$ is even. We do not attempt a careful analysis of this case, but we point out the following

Proposition 4.1. Let $t, b$ be mutually prime odd numbers. Then the basic character of $t$ mod $b$ coincides with the basic character of $t \bmod 2 b$.

Proof: Let the quasi-order of $t \bmod b$ be $n$, and let $t^{n} \equiv \epsilon \bmod b$. Since $t^{n}-\epsilon$ is even, it follows that $t^{n} \equiv \epsilon \bmod 2 b$. It next follows that $n$ is the quasi-order of $t \bmod 2 b$; for the quasi-order of $t \bmod 2 b$ is seen to be neither less than nor greater than the quasi-order of $t \bmod b$. This proves the proposition.

Finally, we analyse the basic character of $t \bmod 2^{n}, n \geq 2$; of course, $t$ is then odd.

Theorem 4.2. Let $d(t)=q \geq 2$. Then the quasi-order of $t \bmod 2^{n}$ is

$$
\begin{array}{ccc}
1 & \text { if } & n \leq q \\
2^{n-q} & \text { if } & n>q
\end{array}
$$


Moreover, $t$ is not basic mod $2^{n}$.

Proof: We have $t=1+c 2^{q}$, with $c$ odd. The conclusion is obvious if $n \leq q$. Let $n>q$. Now since

$$
\left(t^{2^{r-1}}-1\right)\left(t^{2^{r-1}}+1\right)=t^{2^{r}}-1, r \geq 1
$$

it follows by an easy inductive argument on $r$ that

$$
d\left(t^{2^{r}}\right)=q+r, r \geq 0
$$

plainly

$$
t^{2^{r}}+1 \equiv 2 \bmod 4, r \geq 0
$$

Thus

$$
t^{2^{n-4}} \equiv 1 \bmod 2^{n}
$$

while

$$
t^{2^{n-1-1}} \not \equiv \pm 1 \bmod 2^{n}
$$

establishing the theorem.

We can also handle the case $q=1$. Thus let us suppose $d(t)=1$, so that

$$
t=1+2 c, \text { with } c \text { odd. }
$$

We write

$$
t=-1+2^{q^{\prime}} c^{\prime}, \text { with } c^{\prime} \text { odd }
$$

notice that $q^{\prime} \geq 2$.

Theorem 4.3. If $t$ is given by (4.4), with $q^{t} \geq 2$, then

(i) if $n \leq q^{\prime}$, the quasi-order of $\bmod 2^{n}$ is 1 and $t$ is basic;

(ii) if $n>q^{\prime}$, the quasi-order of $t \bmod 2^{n}$ is $2^{n-q^{\prime}}$ and $t$ is not basic.

Proof: (i) is obvious. Thus we suppose $n>q^{\prime}$. As before, we exploit the identity

$$
\left(t^{2^{r-1}}-1\right)\left(t^{2^{r-1}}+1\right)=t^{2^{r}}-1
$$

but now only for $r \geq 2$. For we deduce from (4.4) that $t^{2}=1+2^{q^{\prime}+2} c^{\prime \prime}$, with $c^{\prime \prime}$ odd.

Thus

$$
d\left(t^{2^{r}}\right)=q^{\prime}+r, r \geq 1
$$

and

$$
t^{2^{r}}+1 \equiv 2 \bmod 4, r \geq 1
$$

This shows that

$$
\begin{aligned}
t^{2^{n-q^{\prime}}} & \equiv 1 \bmod 2^{n}, \\
t^{2^{n-q^{\prime}-1}} & \equiv \equiv \pm 1 \bmod 2^{n},
\end{aligned}
$$

establishing the theorem. 


\title{
References
}

1. P. Hilton AND J. Pedersen, Folding regular star polygons and number theory, Math. Intelligencer 7, no. 1 (1983), 15-26.

2. P. HILTON AND J. PEDERSEN, Certain algorithms in the practice of geometry and the theory of numbers, Publ. Sec. Mat. Univ. Autono ma Barcelona 29, no. 1 (1985), 31-64.

3. P. HILTON AND J. PEDERSEN, The general quasi-order algorithm in number theory, Int. Journ. Math. and Math. Sci. 9, no. 2 (1986), 245-252.

4. P. HILTON AND J. PEDERSEN, On the complementary factor in a new congruence algorithm, Int. Journ. Math, and Math. Sci. 10, no. 1 (1987), $113-123$.

5. P. Hilton AND J. PEDERSEN, Geometry in practice and numbers in theory, Monographs in Undergraduate Mathematics 16 (1987), 37.

6. J. FROEMKE AND J.W. Grossman, An algebraic approach to some number-theoretic problems arising from paper-folding regular polygons, American Math. Monthly 95, no. 4 (1988), 289-307.

\author{
P. Hilton: Department of Mathematical Sciences \\ SUNY Binghamton \\ Binghamton, New York 13901 USA \\ J. Hooper: Department of Mathematics \\ University of Utah \\ Salt Lake City, Utah 84112 USA \\ J. Pedersen: Department of Mathematics \\ University of Santa Clara \\ Santa Clara, California 95053 JiSA
}

Rebut el 12 de Setembre de 1988 